\title{
Politique
}

\section{Résolution des conflits et crise de décision}

\section{Julien Bauer}

Numéro 13, printemps 1988

Crise de décision

URI : https://id.erudit.org/iderudit/040582ar

DOI : https://doi.org/10.7202/040582ar

Aller au sommaire du numéro

Éditeur(s)

Société québécoise de science politique

ISSN

0711-608X (imprimé)

1918-6584 (numérique)

Découvrir la revue

Citer cet article

Bauer, J. (1988). Résolution des conflits et crise de décision. Politique, (13), 5-36. https://doi.org/10.7202/040582ar d'utilisation que vous pouvez consulter en ligne.

https://apropos.erudit.org/fr/usagers/politique-dutilisation/ 


\title{
RÉSOLUTION DES CONFLITS ET CRISE DE DÉCISION
}

\author{
Julien Bauer \\ Université du Québec \\ à Montréal
}

Les conflits constituent dans la société une réalité désagréable mais inéluctable. Si l'on se réfere à la définition courante: antagonisme, contestation entre deux puissances ${ }^{1}$, à moins de croire à l'unanimisme et au paradis terrestre, il est difficile de concevoir une société vivante sans conflit. Les auteurs marxistes voient dans une forme exacerbée du conflit, la lutte de classes, le moteur de l'histoire. Les auteurs conservateurs valorisent le maintien d'un certain type de rapports sociaux, par le recours, le cas échéant, à la répression, autre forme de conflit. Les deux écoles s'entendent pour dire que la société n'est pas statique mais est traversée par des conflits. Même ceux qui minimisent l'importance des conflits sociaux dans le développement de la société comme Weber ont été frappés par la permanence des conflits au niveau politique, l'opposition étant inhérente à la politique. Pour reprendre l'expression de Boudon «les conflits ne sont chroniques et endémiques que dans le sous-système politique» ${ }^{2}$.

1. Définition du Robert

2. Boudon, Raymond (1984) La place du désordre. Paris, Presses Universitaires de France.

Politique, 13 (Printemps 1988). 
Antagonisme, contestation, conflit, le système ou le soussystème en conflit, visé par l'antagonisme et la contestation est appelé à prendre des mesures pour répondre aux menaces. Il est amené à prendre des décisions.

Une littérature abondante est disponible sur la prise de décision. Très fréquente est la croyance en l'existence d'une résolution des conflits par des décisions de type scientifique. Elle vise à présenter des décisions raisonnables comme étant rationnelles.

Lancée par Borel et complétée par von Neumann et Morgenstern $^{3}$, la théorie des jeux propose une analyse du comportement dans une conjoncture économique concurrentielle, analyse qui a été étendue à tout système concurrentiel, y compris le système international. Cette théorie ne peut s'appliquer que si tous les joueurs sont d'accord sur l'objectif à atteindre et les moyens légitimes à utiliser. Les stratégies mises sur pied pour résoudre des conflits commerciaux relèvent souvent de ce type d'analyse: l'objectif est d'augmenter la part du marché pour l'entreprise, les moyens sont les prix, le marketing, la publicité etc., le tout encadré par des lois et des règlements qui s'imposent à tous les joueurs (exemple: interdiction de la publicité frauduleuse). Il suffit qu'un des joueurs ne respecte pas les règles du jeu pour que la théorie devienne inopérante, comme a pu le constater Mc Namara durant la guerre du Vietnam.

Plus proches de la réalité sont les théories de la «décompression", à la Kissinger, qui justifient les reculs pour limiter les risques de généralisation des conflits. Il s'agit en fait de masquer l'impuissance des grandes puissances à faire prévaloir en toutes circonstances leurs points de vue. Les règles du jeu sont prévues pour empêcher les risques de dérapage ${ }^{4}$.

3. von Neumann et Morgenstein (1947), Theory of Games and Economic Behaviour. Princeton University Press.

4. L'expression a également été utilisée pour expliquer le laps de temps nécessaire, de nombreuses années, pour que le gouvernement militaire brésilien cède le pouvoir à un système démocratique. 
Mehl voit dans la structure de la décision «un ensemble ternaire où l'on distingue le calculable, l'incertain et l'axiologique» ${ }^{5}$. Appartient au calculable tout ce qui est étudiable et analysable à partir de données, appartient à l'incertain les choix qui ne reposent que partiellement sur les données, l'information étant incomplète; appartient enfin à l'axiologique le choix entre les valeurs qui relève de la morale et non pas du calcul. Selon Mehl il est légitime que les experts tendent à réduire la zone de l'incertain au profit du calculable; par contre, affirmant que «le choix axiologique est l'essence du politique ${ }^{6}$, il repousse les tentatives de calculer ce qui est axiologique.

Toute différente est l'approche des relations humaines dont une des caractéristiques est le rôle de la décision dans la solution des conflits ${ }^{7}$. Pour régler les conflits intra et inter-organisationnels, il faut connaître les motivations des participants, individus et groupes, et prendre les décisions qui amélioreront l'adéquation entre les objectifs des participants et ceux de l'organisation, pour la plus grande gloire de l'organisation. Cette vision volontariste considère la décision comme le coeur de l'organisation, comme l'organe indispensable à la survie de l'organisation. Les conflits sont réductibles à condition que les membres des organisations y mettent du leur et que les structures organisationnelles favorisent la recherche de la solution. Les conflits sont donc perçus comme étant à la fois inévitables et se prêtant à des solutions.

Or membres et structures des organisations constituent des systèmes qui, selon Crozier, ne se prêtent pas à la résolution des conflits. Il soutient que les dysfonctions sont inhérentes à tout système bureaucratique ${ }^{8}$ et qu'elles n'encouragent pas les acteurs

5. Mehl, Lucien (1968), "L'informatique, la connaissance et l'action», Bulletin de l'Institut international d'administration publique. oct-déc, 12-14.

6. Ibid.

7. March, J.-G., et Simon, H. (1969), Les organisations, Paris, Dunod.

8. Crozier, Michel (1964), Le phénomène bureaucratique, Paris, Le Seuil; Crozier, Michel et Friedberg, Erhard (1977), L'acteur et le système, Paris, Le Seuil. 
à modifier les règles du jeu. Il croit cependant que le progrès est inévitable et que des mesures non prévues, des explosions en périodes de crise, sont récupérées par le système. Ce que Crozier appelle des cercles vicieux (centralisation et raisonnement déductif en France, court terme et spécialisation aux États-Unis ${ }^{9}$ ) sont les garants de la continuité du système. La vision de Crozier est optimiste. Son pragmatisme lui fait croire qu'il existe des solutions, ne serait-ce qu'à travers une série de cercles vicieux.

C'est Sfez qui, croyons-nous, a le mieux précisé les fonctions de la décision ${ }^{10}$. Il en retient quatre ${ }^{11}$ mais deux nous apparaissent plus utiles pour notre analyse: permettre à l'auteur d'agir et à l'agi de supporter le monde. D'un côté les responsables politiques, peu importe le processus qui les a porté au pouvoir, se veulent des décideurs, veulent croire qu'ils ont prise sur les évènements, qu'ils peuvent changer le cours des choses. Si leur perception d'eux-mêmes était d'être des objets de l'histoire, de simples bouchons à la superficie de la société, pourquoi chercheraient-ils le pouvoir? D'un autre côté, ceux qui doivent vivre les décisions veulent y croire car elles leur permettent de définir la source de leurs problèmes, les responsables qui ont réussi ou échoué, les héros et les boucs émissaires ${ }^{12}$.

La société est ainsi traversée par des conflits qui obligent les organisations à prendre des décisions pour garder un minimum

9. Crozier, Michel (1980), Le mal américain, Paris, Fayard, p. 261.

10. Sa théorie du surcode lui fait analyser la décision non pas comme une solution à un conflit mais comme porteuse de changement social. Sfez, Lucien (1976) Critique de la décision, Paris, Presses de la Fondation Nationale des Sciences Politiques.

11. Sfez présente ainsi les quatre fonctions de la décision:

1) permettre à l'acteur d'agir;

2) permettre à l'agi, au citoyen, de supporter le monde;

3) fragmenter les actes étatiques en autant de compétences respectives, mais quelques fois concurrentes;

4) séparer l'élaboration de la politique de la prise de décision au niveau opérationnel, Ibid p. 10-2.

12. "Grâce à la décision, le citoyen crée le bouc émissaire", Ibid, p. 11. 
de pouvoir ou, pour reprendre Bourricaud: «la négociation est l'essence du pouvoir" ${ }^{13}$. Le pouvoir ne peut entraîner qu'une remise en question, une contestation, donc un conflit pour aboutir à une nouvelle répartition du pouvoir.

Autant nous estimons utiles les diverses approches dites scientifiques de la décision et de la résolution des conflits - Mehl n'affirme-t-il pas qu'il n'est jamais agréable de prendre une décision dans le brouillard? - autant nous croyons indispensable une certaine méfiance: elles ne fournissent que des outils, non des réponses, des éléments, non un tout. Dans le souci de rejeter les théories globalisantes, Lindblom et son incrémentalisme, sous prétexte de coller à la réalité, en évacuent les valeurs, ce qui appauvrit la compréhension des phénomènes. Sfez, lui, est tellement éclectique, que la décision devient un discours qu'il qualifie de "fantastique». Nous croyons que la complexité du réel est irréductible à une théorie et que l'existence de théories concurrentes, voire contradictoires, une connaissance imparfaite des problèmes, une rationalité limitée des acteurs ne peuvent conduire qu'à des solutions, et des analyses des solutions, elles-mêmes imparfaites, limitées, boîteuses et ambiguës.

Le conflit est une lutte pour le pouvoir; il naît et se développe dans un système, lui même un ensemble organique vivant en équilibre et poursuivant un but, qui peut être local, régional, national, inter ou supranational. Le système ne peut fonctionner qu'avec un minimum de consensus. Nous considérons que le consensus est le plus petit commun dénominateur d'une société. Une diminution du consensus conduit à une montée de la coercition et de la violence légitime (Weber) pour assurer le respect des institutions. Le recours aux sanctions ne peut être qu'un pis-aller car un système politique ne peut vivre sans un minimum de

13. Bourricaud, François (1961), Esquisse d'une théorie de l'autorité, Paris, Plon. 
consensus des éléments constituants. Un tyran peut terroriser le peuple, il ne peut le dissoudre. Le consensus, à son tour, assure la légitimité au système. La légitimité reconnue au système, à ses institutions, à ses organismes dirigeants est l'expression du consensus et limite le recours aux sanctions. Le système, légitime et consensuel, est l'objet de cenflits pour le pouvoir. Ces conflits suivent des règles du jeu acceptées par toutes les parties en concurrence. Cette chaîne qui conduit du conflit aux règles du jeu suppose consensus et légitimité.

Tous les conflits se déroulent-ils selon des règles du jeu reconnues par tous les participants à l'intérieur du système? Une analyse, même sommaire, des conflits nous conduirait à une réponse négative. Nous croyons qu'il est essentiel de distinguer entre les conflits dans le système et les conflits contre le système. Les conflits dans le système naissent et se développent dans un cadre consensuel suivant des règles du jeu. La résolution des conflits est donc possible par l'application des règles qui déterminent les gagnants et les perdants. Les conflits contre le système naissent et se développent dans un cadre différent, marqué par l'absence de légitimité du système, de consensus et donc sans règle du jeu. Il ne s'agit plus de trouver une solution à l'intérieur du système mais de détruire un système pour le remplacer par un autre.

Pour reprendre la terminologie de Popper ${ }^{14}$, les premiers conflits relèvent de lois «conditionnelles» inspirées de théories «scientifiques», les seconds de lois «absolues» inspirées de théories «métaphysiques». En d'autres termes, les conflits dans le système aboutissent à une solution qui est un compromis, un nouvel équilibre entre les participants au système. Les conflits contre le système relèvent d'un absolu, exprimé sous formes de valeurs, croyances, idéologies, philosophies qui constituent, en elles-mêmes,

14. Popper, Karl (1956) Misère de l'bistoricisme. Paris, Plon. 
des systèmes. Dans ce cas, les compromis ou concessions sont perçus comme des compromissions, voire des trahisons. Il ne saurait y avoir de solution au conflit autre que la victoire totale, la Vérité et le Bien triomphant de l'Erreur et du Mal.

Le mythe prométhéen, cher à la pensée occidentale, nous pousse à croire qu'à tout conflit correspond une solution, qu'il n'existe pas de question sans réponse et que si la réponse nous échappe, c'est à nous de faire un effort pour la trouver. Solution d'un conflit signifie qu'une entente, établissant un nouvel équilibre entre les acteurs, est atteinte et que le conflit perd ainsi sa raison d'être. L'entente suppose des règles du jeu qui permettent de formuler une nouvelle donne. Cette vision positiviste des choses nous paraît erronée. Nous suggérons que face à des conflits métaphysiques, il n'existe pas de solution globale et que la recherche à tout prix d'une telle solution globale peut être un ferment de continuation et d'aggravation des conflits. Notre hypothèse selon laquelle la solution pour limiter l'expansion des conflits peut être de refuser les solutions drastiques n'est pas pessimiste mais sobrement réaliste. En l'absence de légitimité, de consensus et de règles du jeu, le conflit continue soit jusqu'à la victoire d'une partie et sinon la disparition du moins l'écrasement de l'autre ${ }^{15}$, soit jusqu'à l'épuisement mutuel où le conflit se calme faute de combattants. Dans l'un ou l'autre cas, le conflit n'a besoin que d'une occasion pour reprendre.

L'absence de légitimité et de consensus ne signifie pas, ipso facto, l'absence de toute règle du jeu mais l'absence de toute règle du jeu de jure. En effet, de telles règles seraient, indirectement, reconnaître la légitimité de l'autre. Par contre, les antagonistes peuvent se reconnaître de fait et appliquer des règles du jeu de facto. Des compromis et des concessions peuvent être atteints de fait, ce qui atténue le conflit, sans être consacrés par un accord

15. symbolisé par l'expression de «reddition sans condition". 
sur les principes, accord innatteignable car on ne saurait transiger sur les questions d'ordre métaphysique.

Les conflits contre le système ne peuvent trouver de solutions permanentes à moins qu'une des parties ne disparaisse. Lorsque les parties se donnent des règles du jeu de facto, les conflits peuvent connaître des périodes d'accalmie, des solutions temporaires. Ces solutions temporaires ont d'autant plus de chance de durer qu'aucune des parties ne perd la face et ne doit renoncer à des croyances, des valeurs, des philosophies, des idéologies profondément ancrées. Imposer une solution qui aille au fond du conflit, qui atteigne le niveau métaphysique, devient alors une recette pour le relancer, non pas seulement au niveau métaphysique où il n'avait jamais vraiment disparu, mais sur le terrain où il ne se manifestait plus que faiblement.

Même pour les conflits dans le système, la meilleure solution peut consister à laisser les règles du jeu s'appliquer ou à les améliorer avec le consentement des parties. Là encore, la tentation demeure de vouloir accélérer la résolution du conflit en courtcircuitant les règles du jeu. Ce type d'intervention, sur une base ad hoc, non assuré d'un accord consensuel, peut être efficace à l'occasion mais ne saurait remplacer l'application des règles du jeu du système.

La résolution des conflits est fonction du type de conflit, dans ou contre le système, et du degré de respect des règles du jeu, de jure et de facto.

L'analyse de quelques exemples, choisis aussi bien dans le domaine de l'administration publique que dans ceux de la politique intérieure ou des relations internationales, dans des pays aux régimes politiques divers nous permettra de vérifier nos hypothèses sur les distinctions entre conflit dans le système et conflit contre le système et sur l'importance des règles du jeu. 


\begin{tabular}{lll}
\hline Conflits & Règles du jeu & Solution \\
$\begin{array}{l}\text { Dans le système: } \\
\text { Consensus }\end{array}$ & Application & Solution acceptée \\
$\begin{array}{l}\text { Légitimité } \\
\text { Règles du jeu }\end{array}$ & $\begin{array}{l}\text { Court-circuit } \\
\text { pour améliorer } \\
\text { solution }\end{array}$ & $\begin{array}{l}\text { Solution } \\
\text { temporaire acceptée }\end{array}$ \\
$\begin{array}{l}\text { Contre le système: } \\
\text { Sans consensus }\end{array}$ & $\begin{array}{l}\text { Continuation du } \\
\text { conflit jusqu'à } \\
\text { la victoire ou } \\
\text { Sans légitimité }\end{array}$ & $\begin{array}{l}\text { Pas de solution } \\
\text { globale ou solution } \\
\text { imposée par la la } \\
\text { disparition d'une } \\
\text { des parties }\end{array}$ \\
$\begin{array}{l}\text { ou avec règles du } \\
\text { jeu de facto }\end{array}$ & $\begin{array}{l}\text { Continuation du } \\
\text { conflit de principe } \\
\text { mais atténuation } \\
\text { du conflit } \\
\text { par compromis et } \\
\text { concessions de } \\
\text { fait }\end{array}$ & Solution ambiguë \\
\hline
\end{tabular}

\section{Les conflits dans le système}

Parmi les cas de conflits dans le système avec application des règles du jeu, nous citerons le débat constitutionnel (référendum et accord du Lac Meech) et certains conflits de travail dans les secteurs public et parapublic au Québec.

La vivacité du débat qui a entouré le référendum de 1980 sur la souveraineté-association a tendance à occulter le fait que, malgré les apparences, il s'agissait bel et bien d'un conflit dans le système avec observance des règles du jeu. Quelques soit la terminologie utilisée, l'objectif du gouvernement péquiste était d'aboutir à un État souverain du Québec lié au Canada par des accords économiques et monétaires. Le but était donc une complète transformation du système mais à l'intérieur de règles du jeu admises de part et d'autre. Le conflit s'est déroulé dans un cadre consensuel où la légitimité des parties et les règles du jeu étaient acceptées comme allant de soi. 
Le fait même qu'un parti politique visant à changer fondamentalement le système soit légal et légitime, témoigne du niveau consensuel de la société. Il suffit d'évoquer l'éventualité d'un parti indépendantiste corse ayant le droit de poser la question sur l'appartenance à la République française ou celle d'un parti indépendantiste en Lithuanie ou en Ukraine, même si la Constitution soviétique reconnaît le droit théorique de se séparer de l'Union, pour se rendre compte de l'étonnant niveau de consensus d'un système qui admet sa remise en cause. D'un autre côté, il est non moins remarquable que les tenants de la souveraineté aient accepté de jouer à l'intérieur de règles, la principale étant la nécessité d'une légitimation démocratique. Les mesures prises par les antagonistes, comités chapeaux pour le OUI et le NON, création d'un organisme fédéral pour promouvoir l'unité canadienne..., avaient bien sûr pour objectif de favoriser l'une ou l'autre option mais les deux parties ont été contraintes, justement parce que le système était consensuel, d'admettre que, d'une façon ou d'une autre, les résultats du référendum s'imposeraient aux deux, que ni l'une ni l'autre ne recoureraient à des moyens non-consensuels, comme la force ou le non respect des lois, pour contourner ces résultats. La profondeur des convictions n'a pas empêché, à quelques bavures près, un conflit sur l'avenir du Canada et du Québec d'une rare civilité. C'est, à notre sens, en fonction du consensus existant dans le système que les règles du jeu ont pu être respectées à ce point par les parties.

Que le Québec n'ait pas signé la Constitution de 1982 a affaibli le consensus et, par conséquent, la légitimité du système. L'accord du Lac Meech ${ }^{16}$, en dehors de ses justifications électoralistes, avait pour objectif de remédier à cette situation, en faisant passer la politique canadienne d'une situation d'exclusion (le Québec étant en dehors) à une situation d'unanimité. Aussi bien pour le

16. dont la ratification se fait attendre. 
rapatriement de la Constitution de 1982 que pour l'accord du Lac Meech, les négociateurs ont travaillé dans une atmosphère de pression, de «c'est maintenant ou jamais». Dans le premier cas, les règles du jeu n'étant pas rigoureusement respectées, le résultat a été jugé, même par ceux qui l'applaudissaient, comme boîteux. L'accord de 1987 , tout unanime qu'il soit, ne manque pas d'incertitude sur la signification réelle du texte, sur la division des pouvoirs entre le gouvernement fédéral et les provinces, en particulier le Québec, sur le sens à donner à la notion de "société distincte», sur la Charte des Droits et Libertés. Les tribunaux détermineront la signification de l'accord. Ce qui nous paraît important dans ce dossier, c'est que l'accord fait passer le conflit de la marge à l'intérieur du système, qu'il est volontairement ambigu pour favoriser le consensus, que cette ambiguïté deviendra source de conflits internes au système.

Un système fortement consensuel impose des règles aux parties en conflit. Le consensus étant jugé indispensable, la solution retenue peut être ambiguë car mieux vaut pour le système une ambiguïté consensuelle (remise par les politiciens du dossier aux tribunaux) que des remises en cause du système, mieux vaut des conflits limités internes que des conflits plus larges avec leurs risques de dérapage, de non-respect des règles du jeu.

Un conflit dans le système qui se déroule selon des règles du jeu précises peut prendre un temps relativement long pour trouver une solution. Dans ce cas la tentation est grande de passer par dessus les règles du jeu pour accélérer le dénouement du conflit. Les parties peuvent décider, de concert, d'accélérer le processus, d'établir des règles du jeu améliorées afin d'accroître les capacités du système à trancher, consensuellement, les conflits. Une autre possibilité est pour l'une des parties de demander un court-circuitage du processus sans obtenir l'accord de l'autre. Une troisième option est pour l'une des parties, en position de force, d'imposer une solution non consensuelle, contraire aux règles du 
jeu. Une quatrième, enfin, consiste à profiter d'une position de force pour introduire des transformations dans les règles du jeu mais de telle façon que le système modifié soit acceptable par toutes les parties.

Le premier cas, celui d'un consensus pour améliorer le processus de résolution des conflits, est observable dans les premières négociations collectives de l'État avec les syndicats des secteurs public et parapublic du Québec. Les règles du jeu des premières négociations prévoyaient qu'elles se dérouleraient à la base, au niveau des syndicats locaux et des employeurs publics locaux, commissions scolaires, hôpitaux etc. La pratique qui vit une suite de conflits de travail, de grèves, de lois spéciales poussa les parties à formuler un nouveau cadre qui centralisait de jure ce qui était déjà centralisé de facto en désignant les centrales syndicales comme agents négociateurs et en imposant un regroupement des employeurs dans les domaines des affaires sociales et de l'éducation ${ }^{17}$. Face à des difficultés d'application, les parties s'entendaient pour introduire des changements aux règles du jeu permettant de limiter les risques de lenteur ou de blocage du système.

Confrontée à une situation qui semble sans issue, une des parties en conflit peut suggérer de contourner les règles pour aboutir à une solution. L'autre partie n'est pas tenue d'accéder à cette demande et peut préférer laisser le processus suivre son cours. C'est ce qui est arrivé pendant le conflit entre le Syndicat des Professeurs de l'Université du Québec à Montréal et l'UQAM ${ }^{18}$. Le syndicat en grève ne voyant pas de solution à l'horizon tenta de profiter de l'élection d'un nouveau gouvernement pour proposer une négociation au sommet, autrement dit un court-circuitage des règles. Lors de sa première conférence comme chef de gouvernement, René Lévesque, interrogé sur ses intentions concernant

17. Loi 95 de 1974

18. 18 octobre 1976-18 février 1977 
le conflit entre le syndicat de professeurs et l'institution universitaire répondit en substance qu'il ne voyait pas le rôle du Premier Ministre comme étant de jouer les pompiers pour éteindre les incendies et que son ministre de l'éducation suivait le dossier. Cette réponse, peu appréciée des grévistes, était cependant fondée. Si le Premier Ministre se sent tenu de s'impliquer directement dans tous les dossiers litigieux, de trouver une solution à tous les conflits, il court le risque de n'être rien d'autre qu'un extincteur et de ne plus avoir le temps de remplir ses obligations de chef du gouvernement. Par contre, le même René Lévesque, voyant le conflit s'éterniser et risquer de devenir un abcès de fixation pour son administration, fit passer le message aux parties dépendantes de lui (Conseil du Trésor, Ministère de l'Éducation, Université du Québec) qu'il était temps de lâcher du lest et d'en arriver à un accord. Ne pas intervenir trop tôt et ouvertement, ne pas accepter un changement aux règles, afin de ne pas encourager les parties en conflit à s'en remettre au Premier Ministre, entrainnant un engorgement des décisions au sommet de la hiérarchie, mais cependant se tenir au courant et intervenir discrètement, sans toucher aux règles du jeu, pour éviter des risques de dérapage nuisibles au gouvernement. Dans ce cas l'intérêt du syndicat était d'aller au-delà des règles, celui de l'État de respecter ces règles pour éviter un précédent.

Si le court-circuitage est négociable, il peut également être imposé. Dans le cas du secteur public, la partie étatique jouit d'un avantage. Elle peut imposer, par voie de législation ou de décret, une solution non négociée, non consensuelle. C'est une telle solution que le gouvernement du Québec imposa à ses salariés en 1982 et 1983. Devant le refus des syndicats d'accepter de revoir à la baisse des conditions de travail dûment négociées, le gouvernement eu recours à une législation violant le cadre consensuel qui avait fonctionné jusque là. La loi 70 de juin 1982 prolongeait de trois mois les conventions collectives qui venaient à expiration 
le 31 décembre 1982 et décidait qu’à partir du $1^{\text {er }}$ avril 1983 les traitements des employés de l'État seraient fixés unilatéralement par le gouvernement ${ }^{19}$. La loi 105 de décembre 1982 déterminait les conditions de travail jusqu'au 31 décembre 1985 mais considérait ces conditions imposées comme constituant des conventions collectives $^{20}$. La négociation pour résoudre un conflit de travail faisait place à la détermination unilatérale de ces conditions. L'opposition syndicale fit long feu car le gouvernement utilisa son pouvoir législatif pour briser tout mouvement de contestation.

En termes techniques, une des parties, le gouvernement, avait gagné, avait imposé sa volonté. En termes politiques, le gouvernement savait qu'il ne pourrait pas répéter ce type de comportement, cette violation des règles du jeu. C'est pourquoi, en 1986-1987, le nouveau gouvernement allait au-delà des textes législatifs et, le 11 décembre 1986, déposait, à l'Assemblée Nationale, de nouvelles offres salariales pour 1987 et 1988, manifestant un désir d'entente négociée, perche saisie par les dirigeants des centrales syndicales. Les deux parties essayaient de reléguer au rayon des mauvais souvenirs la disparition de règles du jeu consensuelles en 1982. Ce n'était pas par grandeur d'âme mais par la conscience qu'un règlement consensuel d'un conflit était, à long terme, pour les deux parties, préférable à un réglement imposé.

Plutôt que de recourir à une solution temporaire par un abus de pouvoir, la partie en position de force peut préférer introduire des modifications aux règles du jeu, modifications qui affectent le rapport de force mais ne touchent pas aux principes. De telles modifications, parce qu'elles n'attaquent pas directement le niveau métaphysique, sont plus facilement acceptables par l'autre partie. C'est ainsi, qu'avec les réticences des parties les plus faibles, de nouvelles règles émergent.

19. Loi 70 , juin 1982 , article 4 .

20. Loi 105 , décembre 1982 , article 9. 
Les relations de travail dans les secteurs public et parapublic québecois nous fournissent, à nouveau, un exemple. Le gouvernement souhaitait supprimer les grandes grèves affectant le système public. Une interdiction pure et simple du droit de grève aurait été non seulement revenir sur les acquis de la Révolution tranquille mais aurait également fait l'unanimité du mouvement syndical contre le gouvernement. De plus elle aurait obligé le gouvernement et l'opinion publique à faire face à un problème de société, à trancher entre des droits souvent contradictoires comme le droit de grève et les droits du public à la santé et à la sécurité. L'objectif était de limiter l'utilisation du droit de grève tout en conservant sa légalité. La loi 72 de juin $1982^{21}$ créait un Conseil des services essentiels pour déterminer la liste des services à maintenir en cas de grève. Elle couvrait les services de santé et les services sociaux mais aussi des services dispensés tant par des entreprises publiques que privées (eau, gaz, électricité, téléphone, transports, enlèvement des ordures ménagères etc.). Le gouvernement profitait de la faiblesse du mouvement syndical et de l'exaspération de l'opinion publique pour amorcer un changement dans les règles du jeu et limiter l'ampleur des conflits. Il ne courtisait pas les risques de chaos en interdisant le droit de grève mais prévoyait des moyens pour rendre la grève inefficace. Un peu plus d'un an plus tard, le gouvernement allait plus loin en interdisant les grèves à moins que les services essentiels ne soient maintenus ${ }^{22}$ mais en conservant, en principe, le droit de grève. En fait de nouvelles règles du jeu,

21. Le titre est révélateur: «Loi modifiant le Code du travail, le Code de procédure civile et d'autres dispositions législatives».

22. La loi de la fonction publique de $\mathbf{1 9 6 5}$ avait bien prévu dans son article 75 que «la grève est interdite à moins que les services essentiels et la façon de les maintenir ne soient déterminés par entente préalable entre les parties ou par décision de la Commission des relations de travail du Québec" mais cet article était resté lettre morte. La même formulation, reprise dans l'article 69 de la loi sur la fonction publique de décembre 1983 et s'appuyant sur l'existence du Conseil des services essentiels, introduit une limitation à l'exercice du droit de grève. 
qui ne remettaient pas en cause le principe du droit de grève mais les modalités de son application, devenaient la norme. Dans la mesure où le mouvement syndical était en position de faiblesse et où le gouvernement avait fait preuve à la fois de fermeté ${ }^{23}$ et de souplesse ${ }^{24}$, les syndicats des secteurs public et parapublic acceptaient le nouveau cadre. Plutôt que d'un court-circuit à effet temporaire, comme les mesures de 1982, il s'agissait d'une nouvelle répartition des cartes qui prenait garde de ne pas porter atteinte aux "grands principes», augmentant d'autant les chances d'un consensus.

Les conflits dans le système trouvent une solution par l'application de règles du jeu. Ces règles peuvent être tellement ancrées que les parties les appliquent de façon quasi-automatique. Lorsque les parties estiment les règles inadéquates, insuffisantes pour permettre de trouver une solution rapide et efficace des conflits, elles s'entendent pour changer ces règles, pour se rencontrer dans la zone du calculable. Si les parties n'arrivent pas à s'entendre sur un nouveau cadre, la plus puissante ou la mieux outillée ce qui est le cas de celle qui dispose du pouvoir législatif - a le choix entre imposer une solution, en dehors des normes reconnues, ou imposer un changement des règles. La violation des normes ne peut être qu'exceptionnelle à moins d'accepter la brisure du consensus. Imposer de nouvelles règles tout en prenant soin de laisser une marge de manoeuvre aux autres parties, dans la zone du calculable et dans celle de l'incertain, et de ne pas remettre en cause leurs croyances, zone de l'axiologique, peut devenir le moyen de faire adopter un nouveau consensus pour répondre aux réalités changeantes.

23. Loi 160 de décembre 1986 prévoyant des mesures d'une sévérité extrême en cas de grève illégale.

24. offres salariales du 11 décembre 1986, cf supra. 


\section{Les conflits internationaux dans et contre le système}

Les conflits internationaux, y compris les conflits armés, peuvent se dérouler dans ou contre le système. Les conflits de type traditionnel se déroulent à l'intérieur du système international; les belligérants reconnaissent la légitimité du système et celle de l'adversaire, ils respectent un consensus concernant les règles du jeu, dans ce cas les règles de la guerre. L'objectif le plus fréquent est de modifier le tracé des frontières en utilisant des moyens acceptés de part et d'autre. Le système international n'a pas réussi à interdire la guerre mais il comprend un certain nombre de conventions qui, à défaut de supprimer les conflits armés, en limitent les dégats. Ces conventions constituent les règles du système international et le cadre consensuel dans lequel ont lieu les conflits. Certaines de ces conventions sont fort anciennes comme celles imposées par l'Église au Moyen-Âge qui faisaient des églises des endroits protégés et des fêtes religieuses des périodes de trève. Les conventions modernes, comme celles de La Haye ou de Genève, cherchent, elles aussi à réglementẹr les guerres. La première Convention de La Haye (1899) en fournit un exemple: interdiction pour cinq ans d'utiliser des projectiles jetés de ballons, interdiction des gaz et des balles à fragmentation, traitement des prisonniers et des blessés, création d'une Cour permanente d'arbitrage, etc. Il ne s'agit pas d'empêcher les guerres mais de minimiser leurs coûts. Ces conventions, qui ne s'imposent qu'aux signataires, n'ont de chance d'être signées et appliquées que lorsqu'elles se limitent à des problèmes d'ordre technique (offrir un recours juridique, interdire certaines armes, interdire les sévices contre les prisonniers et les blessés de guerre etc.). Dès que les conventions visent les principes, et non plus les modalités, elles deviennent inopérantes. Il est instructif de noter les références de plusieurs résolutions des Nations-Unies à l'interdiction d'acquérir des territoires par la force alors que toutes les frontières actuelles, en particulier celles résultant de la Seconde Guerre Mondiale, sont 
l'aboutissement de l'utilisation de la force. Ce type de résolution ne peut rester que lettre morte. La résolution des conflits à l'intérieur du système s'appuie sur des règles consensuelles pratiques.

Les conflits contre le système présentent un problème différent. Les belligérants ne reconnaissent de légitimité ni au système international ni à l'ennemi. En l'absence de consensus et de règles du jeu, le conflit armé a pour objectif la destruction de l'adversaire par tous les moyens. Par contre même en n'accordant qu'une légitimité limitée au système et à l'adversaire, les parties peuvent s'entendre sur des règles de facto. Le but officiel est toujours la destruction de l'autre mais le but officieux est de plus en plus un avantage stratégique par l'utilisation de moyens reconnus de part et d'autre. De nombreux conflits oscillent entre l'absence de règle et l'acceptation de règles de facto. Si la guerre totale et l'anéantissement de l'ennemi sont hors de portée, le recours aux règles de facto fournit un moyen de limiter les pertes.

La guerre entre l'Irak et l'Iran est un cas qui emprunte aux divers modèles exposés plus haut. C'est un conflit entre deux États, deux leaders, deux régimes politiques, deux peuples et deux idéologies. Le conflit interétatique est de type traditionnel, à l'intérieur du système: modifier les frontières par le recours à la force. Le conflit entre deux peuples, forme contemporaine de l'animosité ancestrale entre Arabes et Perses, a des aspects axiologiques mais n'empêche cependant pas la reconnaissance de l'autre, la conscience qu'aucun des deux peuples ne va disparaître. Le conflit entre deux régimes ne peut que restreindre le rôle du système international dans la mesure où les belligérants se donnent le droit d'intervenir dans la détermination de leurs régimes politiques respectifs. Ce droit, qu'ils s'accordent, provient du niveau idéologique, d'une vision métaphysique du monde. Les deux parties ne cherchent donc pas seulement la victoire, ce qui est normal dans une guerre, mais la victoire du Bien sur le Mal, la victoire d'Allah sur Satan, la victoire de l'Arabisme sur la Perse. À ce 
niveau métaphysique, les règles du jeu du système international n'ont plus de sens. Les antagonistes ne reconnaissent le système international que lorsque cela fait leur affaire. Ils considèrent que, dans cette guerre pour le Bien, tous les moyens sont bons, non seulement des pertes en vies humaines considérables mais également des moyens clairement prohibés par des traités internationaux (utilisation d'armes chimiques par l'Irak). Les coûts ont beau être élevés, les résultats limités (quelques kilomètres par-ci par-là), l'absence de consensus, le rejet des règles du jeu expliquent la poursuite des combats. L'épuisement des antagonistes peut conduire à un cessez-le-feu, à des solutions concrètes; leur philosophie leur interdit de pactiser avec un adversaire illégitime. Faut-il s'en remettre à une intervention extérieure pour trouver une solution? Nous croyons que si l'objectif est d'encourager une solution sur le terrain, une intervention du système international, possible plus que plausible, pourrait limiter les dégats: boycott des produits pétroliers des deux pays, embargo sur les armes à destination des belligérants en provenance de tous les pays exportateurs... Par contre, une intervention extérieure allant au fond du conflit, une paix de jure entre les deux parties, une solution globale à un conflit métaphysique nous paraissent relever du fantasme prométhéen.

Un autre exemple de conflit contre le système hésitant entre l'absence de règle et des règles de facto est celui du conflit araboisraélien. On y trouve trois cercles qui vont du général au spécifique et du stratégique au métaphysique: le premier regarde les pays arabes et Israël, le second Jérusalem, le troisième le Mont du Temple. Le premier cercle a présenté pendant longtemps et présente encore en partie des caractéristiques de conflit contre le système, fondées sur la non-reconnaissance absolue d'Israël par les pays arabes. La guerre de 1948-1949 ne s'est pas terminée par un traité de paix où les belligérants se seraient reconnus mutuellement mais par de simples armistices rendus nécessaires par les défaites 
arabes et l'épuisement des deux camps. Il est intéressant de noter que si Israël et l'Égypte, la Jordanie, le Liban et la Syrie ont signé des armistices, l'Irak qui n'avait pas de frontière avec Israël a refusé de signer un tel armistice. En 1967, à nouveau, l'Irak est le seul belligérant à n'avoir pas signé de cessez-le-feu car les forces irakiennes étaient placées sous commandement jordanien permettant ainsi au gouvernement irakien de ne pas avoir le moindre contact officiel avec l'ennemi. Le conflit arabo-israélien est un conflit contre le système, les États arabes, à l'exception de l'Égypte, refusant de reconnaître un système international qui comprend Israël.

Le traité de paix entre l'Égypte et Israël a fait passer le conflit du domaine axiologique à celui de l'incertain et du calculable, les deux parties faisant des concesssions et n'ayant pas conscience de toucher aux grands principes. L'abandon de la ville de Yamit par les Israéliens a été perçu comme un évènement politique, stratégique, touchant une ville construite dans le désert et à laquelle ni les Israéliens ni les Égyptiens n'étaient liés par l'histoire, la religion, les croyances, les émotions. Il s'agissait de délimiter une frontière entre deux États.

Toute différente est la question de Jérusalem car il n'est plus question de stratégie mais de sainteté. Si on peut trouver des compromis dans les conflits politiques, territoriaux, stratégiques, historiques etc., la sainteté d'une ville, telle que comprise par les intéressés, ne saurait souffrir de maquignonnage. Ce n'est pas un hasard si les multiples plans de l'ONU sur le sort de Jérusalem et des Lieux Saints n'ont été acceptés ni par les Arabes ni par les Israéliens ${ }^{25}$. Le choix n'est pas entre une solution globale et la guerre mais entre la guerre et des accords de facto que les antagonistes peuvent accepter.

25. Le Morzellec, Joëlle (1979) La question de Jérusalem devant l'Organisation des Nations-Unies, Bruxelles, Établissement Émile Bruylant. 
La situation devient inextricable sur le Mont du Temple. Les Musulmans réclament l'exclusivité des droits sur ce terrain, les Juifs l'officialisation de leurs liens avec lui. Pour des raisons d'ordre métaphysique mais possédant un extraordinaire potentiel politique, aucune des parties ne peut limiter de jure ses droits sur le Mont du Temple. Aucun gouvernement israélien ne peut renoncer de jure à la souveraineté juive sur le Mont, aucun gouvernement arabe ne peut accepter une souveraineté autre qu'arabe. Dans l'état actuel des choses, la souveraineté théorique appartient à Israël, la juridiction de fait à un organisme arabo-musulman, le Waqf. De facto les Juifs se voient interdire l'accès du Mont par des gardes arabes mais le gouvernement israélien accepte cette situation pour éviter une guerre sainte. Imaginer qu'une solution de jure puisse intervenir ne peut germer que dans l'esprit de ceux qui ne comprennent pas à quel point certains problèmes touchent au coeur des croyances des peuples, touchent au niveau métaphysique. Si certains préferent une guerre totale à une paix partielle, nous croyons que la solution du conflit sur Jérusalem et le Mont du Temple passe soit par une guerre dite sainte soit par un ou des compromis boîteux de facto.

Le dernier exemple de conflit international que nous présenterons est celui entre la Lybie et le Tchad. C'est un mélange de conflit dans et contre le système. Le conflit territorial, en particulier la souveraineté sur la bande d'Aouzou, est un conflit dans le système. Si la Lybie occupe le territoire contesté, mais ne l'a pas annexé de jure, c'est qu'elle reconnaît que son occupation est contraire aux règles internationales et à celles de l'Organisation de I'Unité Africaine. Une guerre d'État à État, même accompagnée jusqu'en 1987 d'une guerre civile, est classique. Ceux qui ne sont pas les premiers concernés, c'est-à-dire les non-Tchadiens, peuvent passer des accords ${ }^{26}$, tracer des lignes imaginaires jusqu'où l'ennemi

26. Accord Khadaffi-Mitterrand 
peut aller ${ }^{27}$, se reconnaître des sphères d'influence, le tout selon les us et coutumes du système international. Par contre aucun traité ne peut masquer la réalité d'un conflit racial, d'une opposition historique entre Arabes et Noirs, l'importance non seulement stratégique mais métaphysique de la première victoire noire sur des envahisseurs depuis des siècles. Dans ce cas, le système international nè peut s'imposer aux antagonistes. Même les méthodes guerrières acceptées par tous cèdent la place à des méthodes originales (rezzous tchadiens). Les Tchadiens se battent à la fois à l'intérieur d'un cadre qui a institutionnalisé les règles de la guerre et contre ce système qui limite leurs chances de victoire. À nouveau il est difficile de concevoir un traité de paix en bonne et due forme entre la Lybie et le Tchad; la solution est-elle d'y rêver ou de favoriser des accords de facto qui limitent les risques de guerre sans prétendre régler le fond du problème?

\section{Du rôle de l'ambiguïté}

Passant des conflits internationaux aux conflits internes, nous avons le cas des sociétés non consensuelles avec des règles du jeu de facto. Lorsqu'existe une opposition entre l'État et la société civile ou le Parti et la société, l'État ou le Parti ont l'avantage car ils possèdent le pouvoir législatif et le monopole de la violence légitime. Par contre ce pouvoir n'est pas absolu; il ne peut s'exercer sans un minimum, même faible, d'acceptation du système par les gouvernés, sans un minimum de consensus. Au niveau axiologique, les détenteurs du pouvoir ne sauraient accepter la légitimité de la contestation, les contestataires étant perçus comme hors système, comme des ennemis. Si les contestataires deviennent assez puissants pour remettre en cause le système, les dirigeants ont le choix entre un changement fondamental ou

27. $17^{\mathrm{e}}$ parallèle 
une répression pour imposer l'obéissance. Si la résistance passive entraîne un mauvais fonctionnement du système, la solution consiste en des tentatives de restaurer des règles du jeu de facto.

La Pologne est un exemple de la nécessité pour le pouvoir, à défaut de consensus dans la société, de faire accepter des règles de facto. Le Parti communiste de Pologne, même s'il existe des petits partis, paysan et démocratique, est dans une situation de parti unique, conformément à l'idéologie marxiste-léniniste. La société civile s'oppose au parti par l'intermédiaire de l'Église et de son idéologie chrétienne puis de Solidarité et de son idéologie syndicaliste-démocratique. Jusqu'à l'apparition de Solidarité régnait un compromis de facto entre le Parti et l'Église, l'Église reconnaissant la suprématie de fait du Parti et ce dernier le rôle de l'Église, aucun des deux ne cherchant à régler le conflit au niveau des valeurs (marxisme et athéisme face au christianisme). Avec Solidarité, on est passé d'une opposition tolérée dans le système à une remise en cause du système. Cette remise en cause, appuyée de façon massive par la population, a obligé les détenteurs du pouvoir à faire un choix: ou bien accepter un nouveau système ou bien recourir aux sanctions. Le premier choix aurait vu le PC polonais ou ce qui en restait, avec l'autorisation du grand frère soviétique, abandonner le mythe de la suprématie du parti, pierre angulaire de la doctrine. Le second, celui retenu, a consisté en un coup d'État militaire du général Jaruzelski avec son cortège d'État d'urgence, d'illégalité de Solidarité, d'arrestations massives etc. Les limites de la répression sont apparues assez rapidement: si les Polonais reperdaient leur droit de parole, ils votaient avec leurs mains en réduisant de plus en plus la production dans le pays. Le pouvoir a été contraint de lâcher du lest, ne serait-ce que pour éviter la faillite.

Il est symptomatique que l'auteur du coup d'État ait créé un Conseil consultatif de cinquante-six membres chargés de lui 
faire des recommandations ${ }^{28}$. Devant ce conseil, Jaruzelski a évoqué le niveau métaphysique et a rappelé les trois fonctions du parti: être le serviteur de la classe ouvrière, l'avant-garde du peuple et jouer le rôle dirigeant dans l'État. Cette litanie n'empêche pas que le fait même de créer un Conseil consultatif est un appel du pied aux Polonais, une façon de renouer le contact avec certaines personnalités non-membres du parti. Ce conseil n'a, comme on peut s'y attendre, aucun pouvoir de jure mais son existence, ses rencontres, la publication des interventions de ses membres témoignent d'une tentative du pouvoir de se rapprocher de la société.

Ni le Parti, ni l'Église, ni dans une moindre mesure Solidarité, ne peuvent renoncer à leurs philosophies et, en ce qui concerne les deux premiers, à leurs monopoles idéologiques; le conflit métaphysique ne peut être réglé. Le Parti, l'Église et des éléments proches de Solidarité ne peuvent éviter d'avoir des contacts au niveau de l'incertain et du calculable; le conflit peut trouver des solutions partielles par des accords de facto. Il s'agit moins de trouver une solution que de minimiser les retombées négatives d'un conflit métaphysique dans une société non-consensuelle.

Conflits dans le système avec des règles du jeu, conflits internationaux dans et contre le système, conflits internes avec deux schèmes de référence métaphysique, il nous reste à voir les conflits internes où la dimension métaphysique est reconnue mais sciemment évacuée pour permettre de trouver une solution en utilisant des règles de facto, détachées de justification de jure.

La planification peut être perçue sous deux angles: l'un, positif, y voit un moyen de maîtriser l'avenir par l'utilisation du contrôle sur plusieurs ou l'ensemble des moyens de production; l'autre, négatif, y voit une limite à l'initiative, à la liberté d'entreprise par un contrôle sur la société. Ces deux approches conduisent à

28. Le texte des interventions à la session du 25 septembre 1987 a été publié dans Roda Narodowa. 
une incompatibilité théorique entre l'économie dirigée et l'économie de marché. Il est exact que la planification impérative, lourde, tatillonne, au lieu d'être un agent de développement, produit une bureaucratie inefficace. Que l'on songe à l'URSS pour s'en convaincre. Il est également exact qu'un marché totalement libre conduit à des inégalités socio-économiques jugées inacceptables par la société. La période de la Dépression en est un exemple. Appliquer sur le terrain l'une des deux visions entraîne soit une politique rigide, écrasante, soit l'anarchie. L'aspect axiologique de la planification apparaît dans le fait que, selon René Grégoire "il est des pays où le mot est encore condamné» ${ }^{29}$ et que dans d'autres pays les slogans font appel aux émotions. En France, la présentation du 6e plan de développement économique et social précise que le premier objectif est «d'assurer à la France la maîtrise de son destin ${ }^{30}$ comme si les États capitalistes sans plan à la française se désintéressaient de leur avenir et comme si un État pouvait «maîtriser son destin», mythe prométhéen s'il en est. Planification et libre marché deviennent des mots d'ordre, des expressions qui ne peuvent entraîner qu'adhésion totale ou rejet absolu. La réalité est, heureusement, plus complexe. Ni planification, ni libre marché ne sont des recettes garantissant le développement économique et social. Les pays à économie planifiée ou bien tolérent une économie parallèle, correctif aux insuffisances du plan central, ou rendent leur planification plus souple, ce qui est reconnaître de facto le rôle du marché. Les pays à économie libérale se donnent des mesures pour encadrer les décisions économiques des acteurs que l'on continue à qualifier d'autonomes, ce qui est reconnaître de facto le rôle de la planification. Si appliquer ce qui est devenu une doctrine crée plus de problèmes

29. Grégoire, René, (1970), "Mutations», Esprit, janvier, p. 159.

30. $\mathrm{VI}^{\mathrm{e}}$ Plan de développement économique et social 1971-1975, Rapport général. Les objectifs généraux et les actions prioritaires du $\mathrm{VI}^{\mathrm{e}}$ Plan et Annexes du rapport général, Programmes d'actions détaillées, (1971), Paris, 10-18, p. 13. 
qu'il n'en résout, la solution consiste à trouver des accomodements, des détours pour régler les problèmes concrets, quitte aux idéologues à prétendre que ces accomodements ne sont pas incompatibles avec la doctrine. L'abandon pur et simple de la planification centralisée sous la houlette du parti communiste en Hongrie aurait entraîné une réaction soviétique et des débats sans fins entre tenants de la planification et tenants du marché. Les références incantatoires à Marx et Lénine n'ont pas empêché une politique économique relativement souple. La solution a consisté à aménager le système de façon concrète et non pas à reconnaître publiquement l'échec du système et son remplacement par un autre. Pour éviter des confrontations inutiles, le cadre de référence a été maintenu; pour éviter la sclérose, le système a de fait considérablement changé. Ce refus de la solution globale, de la métaphysique s'est accompagné de solutions partielles, de facto, calculables qui ont permis à l'économie hongroise de relativement bonnes performances. La dichotomie entre le discours de type religieux et la pratique se trouve également aux États-Unis. Les dirigeants américains ne cessent d'invoquer le libre marché; les chantres de ce marché ne se gênent pourtant pas pour demander et obtenir des interventions de l'État que ce soit pour éliminer ou limiter la concurrence internationale (diverses mesures protectionnistes), sauver une entreprise mal gérée que les lois du marché auraient dû conduire à la faillite (Chrysler), participer par ses commandes à la planification de l'industrie aéronautique. Le rôle, les objectifs, les moyens de la planification sont loin de faire l'unanimité aussi bien dans les économies dirigées que dans les économies libérales. Les oppositions de fonds, la dimension métaphysique sont délibérément délaissées ${ }^{31}$.

31. La postface de M.I. Piskotine, professeur de science administrative à l'Université de Moscou au livre de Gournay, Bernard, Introduction à la science administrative (1978), Paris, Presses de la Fondation Nationale des Sciences Politiques, est un modèle du genre. Elle présente aux lecteurs soviétiques un ouvrage qui, bien qu'écrit par un auteur «bourgeois», a "une véritable valeur scientifique». Elle cite Lénine pour bien faire la distinction entre 
Au mieux on salue la doctrine, en fait on cherche des solutions pratiques, acceptables par tous.

Les problèmes de société qui ne portent pas sur le pouvoir et sa répartition (cadre constitutionnel, planification, syndicalisme etc.) mais sur la perception d'elle-même qu'a la société, sur son idéologie, sur ses croyances, sur ses notions de Bien et de Mal trouvent difficilement une solution. Or c'est à de tels problèmes que sont confrontés la société et l'État quand il faut prendre des décisions sur la peine capitale, l'euthanasie, l'avortement etc. Dans un système politique consensuel où s'opposent des systèmes idéologiques, une solution politique doit être trouvée à un conflit sur les valeurs. Les règles du jeu consensuelles, suffisantes pour les autres conflits, peuvent se révéler incapables d'atteindre une solution consensuelle. Une législation, votée démocratiquement par le Parlement, s'avère suffisamment légitime pour que même ses opposants la respectent. Une législation touchant à des croyances fondamentales risque de ne pas être reconnue comme légitime si elle suit la simple arithmétique parlementaire, si elle ne jouit que d'une majorité faible. Selon Lijphart ${ }^{32}$, plutôt que de créer des minorités irréductibles avec ceux qui ne peuvent accepter la législation, minorités se plaçant hors du système et hors du consensus, la "consociational democracy" va plus loin que le respect des règles du jeu et cherche à tenir compte, tout en respectant les vœux de la majorité, des croyances de la minorité. Pour trancher ces conflits sur les valeurs, le calculable peut grignoter l'incertain, il est cependant soumis à l'axiologique. C'est pourquoi ces décisions sont particulièrement difficiles à prendre et politiquement dangereuses pour les gouvernants.

administrations marxiste et bourgeoise mais conclut que le livre «soulève la plupart des problèmes qui viennent à l'esprit lorsqu'on examine l'appareil gouvernemental et administratif». En fait la postface favorise une approche fonctionnaliste sous la protection de références à la doctrine marxiste.

32. Lijphart, Arend (ed), (1969), Politics in Europe. Comparisons and Interpretations, Englewood Cliffs, Prentice-Hall, pp 45-80. 
Les débats sur la peine de mort sont éloquents à ce sujet. Les tenants et les opposants de la peine capitale ont beau citer des études pour étayer leurs positions, ces études ne sont guère convaincantes pour ceux qui croient profondément que la peine de mort doit être maintenue ou abolie au nom de raisonnements ontologiques. Dans l'espoir de l'établissement d'un relatif consensus, la solution peut n'être que temporaire: ne pas changer la loi, pour éviter l'aggravation du conflit, mais également ne pas appliquer la loi. Garder la peine capitale dans les statuts satisfait ses partisans, ne pas l'appliquer satisfait ses opposants. C'est la démarche habituellement suivie par les pays qui, après une période d'ambiguité, ont aboli la peine de mort. Le même cheminement est discernable dans le cas de l'avortement. Au lieu d'établir clairement une législation dans l'un ou l'autre sens, de proclamer leur attachement à l'une ou l'autre philosophie, les pouvoirs publics s'en remettent à un compromis de facto. La loi reste telle quelle mais les contrevenants ne sont pas sanctionnés. Ce qui ressemble à un manque de courage de la part des dirigeants est peut-être une marque plus ou moins consciente de sagesse: un changement de jure qui aurait suscité des oppositions irréductibles à un moment donné peut devenir plus aisément acceptable, même par ceux qui lui sont hostiles, après une période de changement de facto. Vouloir à tout prix officialiser le changement, régler le conflit est un des meilleurs moyens pour relancer le conflit et mettre en péril les modifications déjà acquises.

Le conflit sur l'euthanasie met, lui aussi, face à face deux positions de principe: le droit à la vie jusque et y compris le maintien en vie même sans espoir de guérison et au prix de souffrances et le droit à la mort dans les conditions voulues par le patient. Ce choix auquel se trouvent confrontés quotidiennement malades, médecins, familles, gardiens etc. est à proprement parler axiologique. Résoudre le conflit en faveur de l'une ou l'autre des options risque non seulement d'enflammer les passions, rendant 
difficile un consensus, mais d'aboutir à des conséquences difficilement tolérables: imposer des souffrances inutiles à ceux qui les refusent ou tuer ceux qui ne sont plus en état de se défendre. On comprend que personne ne veuille prendre la responsabilité de formuler une solution. Les réponses actuelles se présentent sous trois aspects:

1. faire sortir la discussion, dans la mesure du possible, de la zone de l'axiologique (définitions techniques de la mort selon l'arrêt de fonctionnement d'un organe, coeur, cerveau, etc);

2. s'en remettre aux tribunaux pour qu'ils créent des précédents sans intervention législative;

3. laisser les médecins décider du sort des malades en fonction, entre autres, des équipements disponibles.

Ces trois réponses ne constituent pas une solution claire. On continue à laisser planer un doute, une ambiguïté qui permet de tenir compte des deux positions.

Une société consensuelle qui doit prendre une décision sur un sujet mettant en cause des croyances fondamentales de divers segments de la population doit concilier le respect des règles du jeu de jure avec le respect de croyances métaphysiques. Cela rend l'utilisation des règles du jeu plus limitée que pour des décisions concernant la répartition du pouvoir. Le caractère absolu des croyances est une entrave à l'acceptation de décisions même lorsqu'elles ont été prises selon les règles du jeu reconnues. Une décision catégorique risque de mettre en danger le consensus ${ }^{33}$.

33. Lors de la discussion au Comité judiciaire du Sénat américain concernant la nomination du juge Bork à la Cour Suprême, le sénateur De Concini a clairement indiqué que la recherche du consensus dans une société de concurrence l'emportait sur la rigueur de la Constitution. "Judge Bork views the Constitution as a bloodless and sterile contract instead of a bond between diverse and competing people to use common sense and consideration of each other as a framework to build a nation". New York Times, 6 octobre 1987. 
Une décision délibérément ambiguë, qui n'attaque pas de front les croyances mais se limite à des solutions partielles, fournit à la société une période d'ajustement et, le cas échéant, la possibilité d'une solution consensuelle.

\section{Conclusion}

Notre objectif n'est pas de nier l'importance de la décision ni d'encourager la non-prise de décision, ce qui constituerait en soi une décision, mais d'éviter de tomber dans le piège de la mystification de la décision, comme si décider était suffisant pour résoudre tous les conflits, quelle que soit leur nature.

Il peut sembler rationnel de vouloir résoudre les conflits, il est souvent raisonnable de limiter les ambitions des preneurs de décision. La sagesse populaire ne dit-elle pas que «le mieux est l'ennemi du bien»? Plutôt que de chercher la solution rationnelle, rationnelle en tout cas aux yeux des décideurs et des observateurs, et de l'imposer aux parties récalcitrantes, avec des chances de succès limitées, nous croyons plus efficace d'aider à faire glisser les conflits d'une catégorie à l'autre, de conflit contre le système à conflit dans le système, de conflit sans règle du jeu à conflit avec règles du jeu de facto, de conflit avec intervention massive et même intempestive à conflit se déroulant selon les étapes prévues. Une telle approche est sans doute moins valorisante pour les décideurs mais possède un potentiel de calmer les antagonismes.

On peut se demander si l'ego des décideurs, ou des apprentisdécideurs, individus et organismes, n'est pas tenu pour plus digne de considération que la solution des conflits. Lorsqu'on réussit à faire glisser un conflit du métaphysique à l'incertain et au calculable, certains s'y opposent. Ils sont les tenants de la solution parfaite, idéale, ils repoussent les compromis atteints au nom de solutions globales dont on sait qu'elles constituent un objectif quasiirréalisable. Les accords de Camp David, qui ont fait passé une 
partie du conflit arabo-israélien de conflit contre le système à conflit dans le système, ont été critiqués par ceux qui aimeraient être les grands maîtres de la solution et se refusent à limiter leurs ambitions à être des artisans de solutions partielles. À les entendre, mieux vaut une bonne guerre qu'une paix limitée... Dans le même ordre d'idée, la loi des services essentiels qui limite le droit de grève dans les services publics, a été critiqué par ceux qui auraient préféré une interdiction pure et simple du droit de grève dans les services publics, comme si une telle loi, et les inévitables conflits qu'elle aurait entraînés, eut été préférable à l'acceptation, même rétive, de nouvelles règles du jeu. À la limite, même lorsque les conflits se déroulent dans un système aux règles du jeu précises, il peut être recommandé de ne pas lui trouver de solution. «La sagesse consiste parfois à laisser sans solution un conflit ${ }^{34}$.

Les conflits, nous l'avons relevé dans l'introduction, sont inhérents à la vie politique. Selon qu'ils se déroulent à l'intérieur ou contre le système, avec ou sans règle de jure et de facto, les résoudre devient un objectif plus ou moins réaliste.

Ce n'est que dans des cas exceptionnels, où une partie menace l'existence même des autres parties par la violation systématique de toutes les règles, que le conflit ne saurait trouver de solution autre que la mise au pas, voire la destruction de la menace. Il n'est alors pas question des finalités et des modalités de la décision mais de l'élimination urgente d'un danger mortel. La majorité des conflits, même armés, se prêtent, eux, à des solutions. Nous avons exposé les raisons qui font que, dans les conflits contre le système et sans règle du jeu, la meilleure approche pour résoudre ces conflits n'était ni la quête ni l'imposition de la solution mais la promotion de modalités pour faire passer les conflits d'une

34. Gournay, op. cit., p. 210. 
catégorie où ils sont quasi-insolubles à une catégorie où des solutions partielles sont plus susceptibles d'être acceptées.

Toute décision pour régler un conflit porte en elle-même les germes des contestations, des conflits à venir. Puisqu'aucune décision n'arrête le cours de l'histoire, imaginer une solution qui résoudrait de façon définitive un conflit, c'est prendre Prométhée pour un prophète. Une grande solution étant non seulement difficile à trouver mais aussi aléatoire, nous croyons qu'un des procédés pour maintenir les conflits dans des limites jugées raisonnables par les parties est de ne pas hésiter à jouer de l'ambiguïté. La principale vertu de l'ambiguïté est de favoriser l'émergence de règles du jeu. La principale utilité des règles est de conserver des canaux de communications indispensables, dans l'avenir, pour toute recherche sinon d'une solution du moins d'un accord. 